\title{
Device Characteristics of Polythiophene-based Field-effect Transistors Fabricated under Various Conditions
}

\author{
Satoshi Hoshino, Manabu Yoshida, Sei Uemura, Takehito Kodzasa and Toshihide Kamata \\ Photonics Research Institute, National Institute of Advanced Industrial Science and Technology (AIST) \\ 1-1-1 Higashi, Tsukuba, Ibaraki 305-8565, Japan \\ s.hoshino@aist.go.jp
}

\begin{abstract}
We investigated the device characteristics of field-effect transistors (FETs) based on a regioregular polythiophene for their dependence on device fabrication conditions. The device performance varied depending on solvents, chemical species of insulator-surface modifier and polymer solution concentration, with the result that the field-effect mobility for hole ranged from $10^{-4}$ to $\sim 10^{-2} \mathrm{~cm}^{2} / \mathrm{V} \cdot \mathrm{sec}$. We concluded from the variation in the device characteristics of a series of FETs made with toluene solutions that, in addition to the higher regioregularity of the polymer, chemical and/or physical properties of the contact between the polymer solution and insulator surface substantially influenced the device performance.
\end{abstract}

Keywords: field-effect transistors, regioregular polythiophenes, field-effect mobility

\section{Introduction}

Organic field-effect transistors (FETs) have attracted a lot of attention due to their recent marked progress in the device performance.[1] Organic semiconductors have a great advantage as regards processability at moderate temperature over typical inorganic counterparts. This material property inspires to fabricate organic FETs on a wide variety of substrates including plastics. There has been a growing interest in organic thin-film transistor circuits on plastics; they are expected to play a role of backplane that enables "flexible" information displays based on liquid crystals or organic light-emitting diodes.

Soluble $\pi$-conjugated polymers have potential for low-cost manufacturing of such organic FET devices by means of solution-based technologies. Regioregular poly(3- $n$-hexylthiophen) (rr-P3HT) has been intensively studied since certain pioneering studies reported an observation of relatively high field-effect mobility $\left(\mu_{\text {fet }}\right)$ for hole $\left(>0.05 \mathrm{~cm}^{2} / \mathrm{V} \cdot \mathrm{sec}\right)$ from FETs prepared on a $c-\mathrm{Si} / \mathrm{SiO}_{2}$ substrate.[2],[3] Owing to the regularly aligned hexyl groups along the polymer backbone, rr-P3HT tends to form highly ordered microcrystalline lamellae in the film formation process from solutions. The superior hole mobility to those generally observed for the other $\pi$-conjugated polymer-based FETs has been explained by the efficient hole transport through the ordered crystalline moiety in the active layer of the FETs.[4] The discovery of the incredible FET characteristics has stimulated a number of rr-P3HT-based FET studies aiming for confirming reproducibility of the efficient device operation and/or gaining further improved performance. [5-9] These recent studies, however, let us to realize the fact that the $\mu_{\text {et }}$ values showed a marked dispersion ranging from $10^{-4}$ to $\sim 0.2 \mathrm{~cm}^{2} / \mathrm{V} \cdot \mathrm{sec}$ although there was no significant difference in the basic device configuration among these FETs. Such possible reasons as degree of polymer purification, production lot of commercial samples, active layer preparation method and molecular weight of the polymer have been proposed for the successful or unsuccessful observations about the $\mu_{\text {et }} \cdot[5-7]$ These results suggest that the higher main chain regioregularity and concomitant self-organization property are not the unique determinant of the efficient device operation, and that numerous factors with regard to device fabrication conditions should influence the device characteristics of the 
rr-P3HT-based FETs as well.

In this paper, we investigated rr-P3HT-based FETs for dependence of the device characteristics on device fabrication conditions such as gate insulator surface modification, cast solvent, solution concentration and environment for the active layer preparation. We found that the device performance depended on these fabrication parameters, by which the $\mu_{f e t}$ was varied from $10^{-4}$ to $-10^{-2} \mathrm{~cm}^{2} / \mathrm{V} \cdot \mathrm{sec}$. We found that the $\mu_{\text {fet }}$ was affected by the property of the solution/insulator surface contact, at which certain interactions between the polymer and surface chemical species played crucial role for determining the morphology of the carrier conduction channel of the FET.

\section{Experiments}

Fig. 1 shows the chemical structure of rr-P3HT and basic device structure of the FETs used in this study. The polymer sample was prepared from commercial rr-P3HT (Aldrich) as follows. First we collected a chloroform-soluble part from as-received polymer. Then we washed the precipitated polymer with boiling hexane and methanol for several hours by soxhlet extraction method. The polymer sample obtained in this way had a weight average molecular weigh of 54000 with a polydispersity index (PDI) of $\sim 1.8$ (based on polystyrene standard). Regioregularity (head-to-tail linkage content) was determined to be more than $96 \%$ from ${ }^{1} \mathrm{H}-\mathrm{NMR}$ analysis.

The FETs used in this study had a bottom-contact configuration with respect to source and drain electrodes. We fabricated the FET on an $n$-doped $\mathrm{Si}$ wafer with a $200 \mathrm{~nm}$-thick thermally grown oxide layer, which functioned as a gate electrode and a gate insulator, respectively. The wafer was ultrasonically cleaned in dichloromethane, $i$-propanol, and subsequently de-ionized water, and dried by $\mathrm{N}_{2}$ blow. We evaporated $\mathrm{Cr}$ (adhesion layer, $<1 \mathrm{~nm}$ ) and $\mathrm{Au}(35$ $\mathrm{nm}$ ) on the $\mathrm{SiO}_{2}$ surface through a metal shadow mask at a pressure of around $10^{-4} \mathrm{~Pa}$, and formed source and drain electrodes consisting of a pair of $100 \mu \mathrm{m}$ wide and $500 \mu \mathrm{m}$ long stripes separated by a $20 \mu \mathrm{m}$ gap. The latter two correspond to the channel width $(W)$ and channel length $(L)$ of the FETs, respectively. The substrate was cleaned by immersing in $\mathrm{H}_{2} \mathrm{O}-\mathrm{H}_{2} \mathrm{O}_{2}-\mathrm{NH}_{4} \mathrm{OH} \quad(5: 1: 1)$ mixture heated at $70{ }^{\circ} \mathrm{C}$ for $10 \mathrm{~min}$ and washed with de-ionized water, then dried in $\mathrm{N}_{2}$ stream prior to chemical modification of the insulator surface.

We used 1,1,1,3,3,3-hexamethyldisilazane

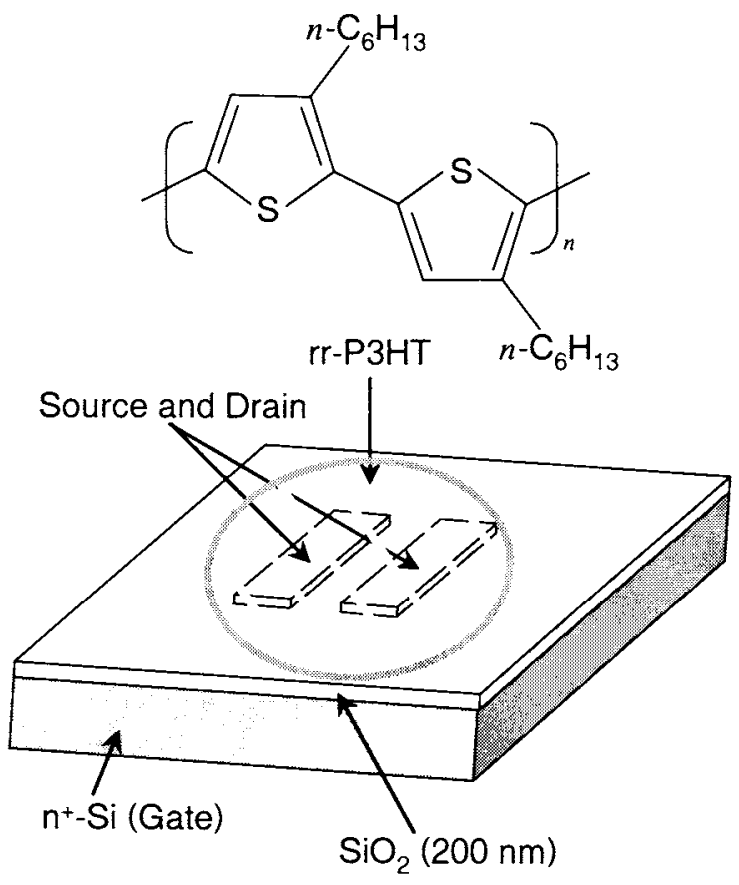

Fig. 1. Device structure of bottom-contact FETs used in this study and chemical structure of rr-P3HT.

(HMDS) and $n$-octyltrichlorosilane (OTS) for the surface modifying reagents. The substrate was soaked in an anhydrous dichloromethane solution of HMDS (20 vol \%) or OTS $(-1.2 \mathrm{mM})$ for $3 \mathrm{~h}$ and then washed with a copious amount of dichloromethane. For the HMDS modification, the solution was heated at $70^{\circ} \mathrm{C}$ during the reaction in a sealed PTFE bottle. The rr-P3HT active layer was cast on the source and drain electrodes from a chloroform or toluene (WAKO, $\infty$ pure grade) solution using a microsyringe. We performed these series of operations in $\mathrm{N}_{2}$ replaced glove box unless otherwise noted.

The current-voltage profile of the FET was recorded in a vacuum (typically $10^{-2} \sim 10^{-3} \mathrm{~Pa}$ ) with KEITHLEY 6430 and 2400 source meters in accordance with a previously reported method.[10]

\section{Results and Discussion}

First we investigated device characteristics of the FET which was fabricated according to the method reported in the seminal studies (FET-1). Briefly, the surface of the gate insulator $\mathrm{SiO}_{2}$ was modified with HMDS and the active layer was prepared from a dilute $(0.05 \mathrm{wt} \%)$ chloroform solution. Fig. 2 shows the drain current-drain voltage $\left(I_{d}-V_{d}\right) \quad$ characteristics (output characteristics) of FET-1 measured with various 


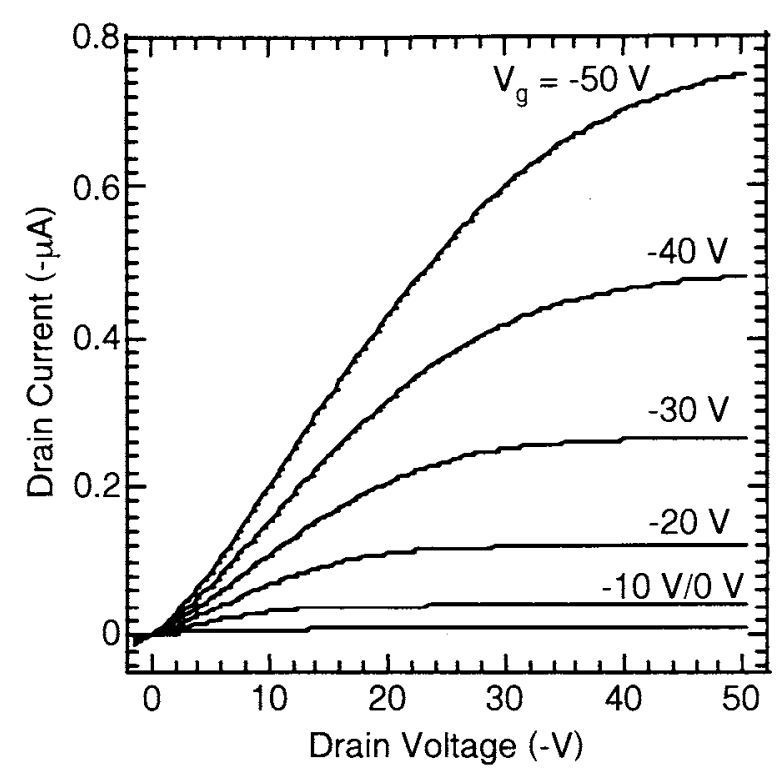

Fig. 2. Output characteristics of FET-1 at room temperature.

gate voltages $\left(V_{g}\right.$ 's). The output characteristics demonstrate that the output current with a negative sign underwent a modulation from the change in the negative gate bias. This means that the predominant charge carriers of the output current are hole and the FET operates in a $p$-channel enhancement mode. As regards the $I_{d}-V_{d}$ profile, the output $I_{d}$ first increased almost linearly and then leveled off with respect to the increase in source-drain electric field. This current-voltage behavior indicates that the hole conduction was regulated by a channel pinch-off originating from a hole depletion region, which gradually expands around the drain electrode as increasing the source-drain bias. We thus employed a simple device operation model based on the gradual channel approximation for analysis of the device characteristics.[1],[11] In this device operation regime, $\mu_{f e t}$ is correlated with dependence of field-effect modulation of $I_{d}$ at the saturation region on $V_{g}^{2}$ as expressed by the following equation,

$$
I_{d}=\left(W C_{i} / 2 L\right) \cdot \mu_{f e t} \cdot\left(V_{g}-V_{o}\right)^{2}
$$

where $W, C_{i}, L$. and $V_{0}$ denote channel width, capacitance per unit area of gate insulator, channel length and threshold voltage, respectively.[11] Fig. 3 shows the $V_{g}$ dependence of the $I_{d}$ at the saturation region (transfer characteristics) together with that plotted for $\left|I_{d}\right|^{1 / 2}$. With the exception of the low $V_{\mathrm{g}}$ region, we confirmed a linear relationship between $\left.U_{d}\right|^{1 / 2}$ and $V_{g}$, suggesting that the FET worked approximately obeying the operation model. The $\mu_{\text {fet }}$ obtained from the transfer characteristics was $1.3 \times 10^{-3} \mathrm{~cm}^{2} / \mathrm{V} \cdot \mathrm{sec}$, which is less than one tenth of that reported by Sirringhaus et al. for a bottom contact device.[3],[4] One possible factor for observing the lower $\mu_{\text {et }}$ is the difference in the molecular weight of rr-P3HT sample used for the experiment; the higher $M_{w}$ and greater PDI of our polymer sample than theirs $\left(M_{w}=\sim 24000, \mathrm{PDI}=1.4\right)$ may prevent sufficient growth of the ordered crystalline lamellae in the active layer during the condensation process from solution. It should be also noted here that, as regards the $\mu_{f e t}$ calculation, Sirringhaus et al. employed another device operation model that took account of a change in the hole-conducting electronic states with applied $V_{g}$.[12],[13] In this device operation regime, $\mu_{\text {fet }}$ is regarded as a $V_{g}$ dependent parameter, and tends to increases as escalating the applied gate bias. The reported $\mu_{e t}$ s were derived mostly from the $I_{d} V_{d}$ characteristics at relatively high $V_{g}$ 's (typically $>70 \mathrm{~V}$ ). In this respect, we may, to some extent, underestimate the $\mu_{\text {fet }}$ value of the FET although the device operation model represented by equation (1) has been widely used for the analysis of organic FET characteristics.[1]

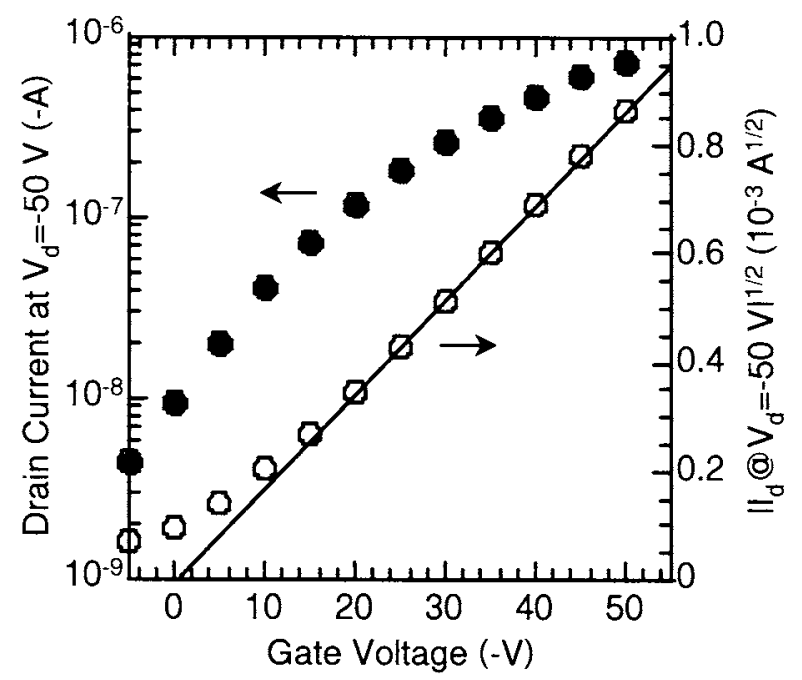

Fig. 3. Transfer characteristics of FET-1 obtained from the $V_{g}$ dependence of $I_{d}$ at $V_{d}=-50 \mathrm{~V}$. 




Fig. 4. Output characteristics of FET-2 at room temperature.

We investigated dependence of the device characteristics on chemical species of the insulator surface and cast solvents and observed a markedly improved $\mu_{\text {fet }}\left(>10^{-2} \mathrm{~cm}^{2} / \mathrm{V} \cdot \mathrm{sec}\right)$ for FET-2, where OTS and a dilute toluene solution $(0.08 \mathrm{wt} \%)$ were used for the surface modifier and active layer cast solution, respectively. Fig. 4 shows the output and transfer characteristics of FET-2. FET-2 yielded larger output current under the hole accumulation regime $\left(V_{g}<0 \mathrm{~V}\right)$ than FET-1 at the equivalent operating condition although there was no marked difference in the off-current (we defined it as $I_{d}$ 's at $V_{g}=0 \mathrm{~V}$ here). This implies that the field-effect modulation of the hole conduction occurred more efficiently in FET-2. From the transfer characteristics shown Fig. 5, we obtained a $\mu_{\text {fet }}$ of $1.5 \times 10^{-2} \mathrm{~cm}^{2} / \mathrm{V} \cdot \mathrm{sec}$, which compares to the typical topmost value recently reported from several research groups.[7],[8],[14] The use of toluene as the casting solvent accounts for, to some degree, the improvement in the device performance; it is likely that the slower evaporation rate than chloroform acted effectively for efficient ordered crystalline lamellae formation, and consequently the hole transport efficiency in the active layer had to be improved.

We revealed, however, that the device characteristics of the FETs fabricated using toluene solutions showed a clear dependence on the chemical species of the insulator surface or solution concentration. Fig. 5 shows the transfer characteristics $\left(\left.I_{d}\right|^{1 / 2}\right.$ plot against $\left.V_{g}\right)$ of FET-3, where active layer was prepared from the same toluene solution as used for FET-2 but the insulator surface was modified with HMDS instead of OTS. Compared with the case of FET-2, the gentle slope observed for the $\left|I_{d}\right|^{1 / 2} \propto V_{g}$ relationship is indicative of the fact that the substitution of the surface modifier adversely affect the device performance, which fact resulted in almost an order decrease of $\mu_{\text {fet }}\left(1.3 \times 10^{-3} \mathrm{~cm}^{2} / \mathrm{V} \cdot \mathrm{sec}\right)$.

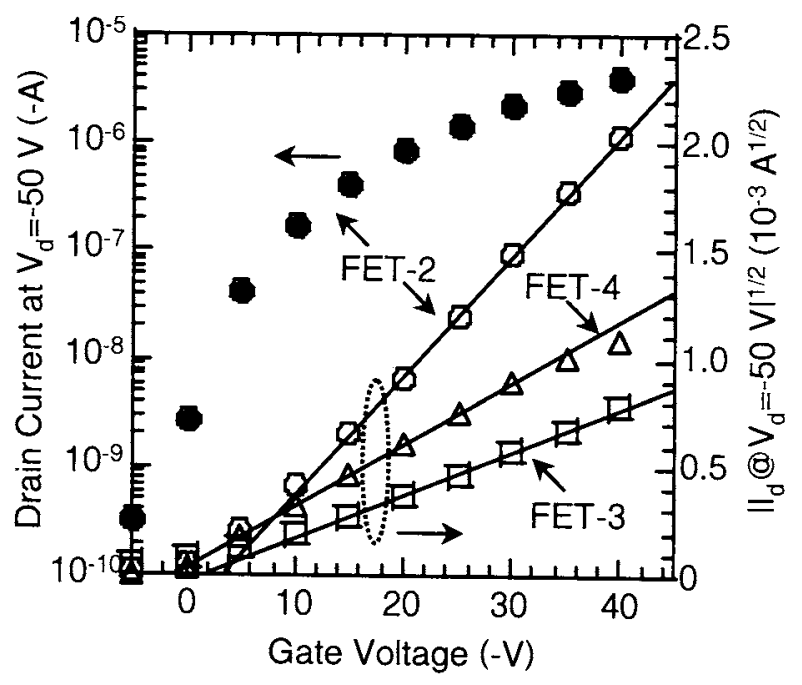

Fig. 5. Transfer characteristics of FET-2 (open and filled circles), FET-3 (open squares) and FET-4 (open triangles) obtained from the $V_{g}$ dependence of $I_{d}$ at $V_{s}=-50 \mathrm{~V}$.

We investigated the dependence on the solution concentration for FET-4, whose active layer was fabricated on the OTS modified surface from a $0.16 \mathrm{wt} \%$ toluene solution. As confirmed from the transfer characteristics found between those of FET-2 and -3 (see Fig. 5), the use of the solution with twice higher concentration was fairly detrimental to the device performance, and the $\mu_{\text {fet }}$ was reduced to $3.9 \times 10^{-3} \mathrm{~cm}^{2} / \mathrm{V} \cdot \mathrm{s}$. As regards the film formation process from these toluene solutions, it is reasonable to conclude that the solidification occurs from the most outer surface of the solution droplet that covered the whole of source/drain electrodes and carrier conduction channel region. Taking into account the facts that solvent (toluene) evaporation occurs at a modestly slow rate, a series of these FETs must be almost the same with respect to the fundamental thin-film 
structure of the active layer in the bulk region. By contrast, the solid-state structure in the vicinity of the gate insulator surface, which part does function as a carrier conduction channel during FET operation, is possibly affected by certain chemical and/or physical properties of the solution/insulator surface contact. We thus conclude that the change of the surface modifiers and solution concentration altered the contact properties, and consequently these FETs had different morphology in the conduction channel region.

In FET-2, interdigitation or quasi-two dimensional crystalline chain packing probably occurred between the surface octyl groups and hexyl side chains of rr-P3HT, and promoted the edge-on orientation of main chain and/or microcrystalline lamellae at the solution-gate insulator interface in the course of the evaporation process. Owing to this anchoring effect, the main chain $\pi-\pi$ stacking was apt to occur parallel to the surface in the vicinity of the gate insulator. We speculate that hole conduction from the source to drain electrodes occurred efficiently through the $\pi$-conjugated plane stacks orienting advantageously for carrier transport, and consequently FET-2 exhibited the improved device performance.

By contrast, in the FETs with the HMDS surface modification, we cannot expect such firm anchoring effect because the gate insulator surface was covered with trimetylsilyl groups by the silylating reagent. It is likely that the conjugated main chain stacks should be oriented, to a certain extent, randomly with respect to the direction to the insulator surface. Thus the noticeable deterioration in the device performance observed for FET-3 may be due to inefficient hole transport in the conduction channel in which the main chain $\pi$-stacks of rr-P3HT had poor in-plane alignment. We found similar degeneration of the device characteristics accompanied by the shortening of alkyl chain length of the surface chemical spices from an FET where ethyltrichlorosilane was used for the surface modifier. Above results suggest that surface chemical species with certain alkyl chain length is necessary for establishing the advantageous thin-film structure in the conduction channel for hole transport.

If the solution concentration is increased, non-specific adsorption of $\mathrm{rr}-\mathrm{P} 3 \mathrm{HT}$ molecules is likely encouraged during the evaporation process in parallel with the interchain interaction between the surface and polymer alkyl groups. In the conduction channel of FET-4, it is likely that the parallel orientation of the main chain $\pi$-stacks was, to some extent, disrupted by the non-specific polymer-surface interaction. We therefore estimate that the moderately degenerated device performance shown by FET-4 is responsible for the conduction channel with modestly inferior morphology for the effective hole conduction to that of FET-2. Finally we report that the device performance of FET-2 was significantly lowered and the $\mu_{f e t}$ was reduced to $<10^{-3} \mathrm{~cm}^{2} / \mathrm{V} \cdot \mathrm{sec}$ if the active layer was prepared in ambient atmosphere. We estimate that the possible anchoring interaction that induces the effective thin-film morphology for hole conduction is seriously inhibited by the adsorption of water molecules on the OTS treated surface.

\section{Conclusions}

We investigated the device characteristics of the rr-P3HT-based bottom-contact FETs for their dependence on the fabrication conditions. The device performance evaluated from the $\mu_{f e t}$ values varied depending on the solvents, chemical species of insulator-surface modifier and rr-P3HT solution concentration. We concluded that such aspects of chemical and physical properties of the contact between the solution and insulator surface as interaction between the rr-P3HT side chain and surface alkyl group and adsorption behavior of the polymer molecules played substantial role for determining the sold-state morphology of the conduction channel and the hole conduction efficiency.

The highest $\mu_{f e t}$ obtained in this study remained to be in the level of $-10^{-2} \mathrm{~cm}^{2} / \mathrm{V} \cdot \mathrm{sec}$, which observation, however, approximately replicated those recently reported by several research groups. We, therefore, speculate that, other than the solid-state structure of the conduction channel, there are several hidden factors in such efficient device operation exhibiting a $\mu_{\text {fet }}$ of more than $10^{-1}$ $\mathrm{cm}^{2} / \mathrm{V} \cdot \mathrm{sec} .[3],[6]$

\section{Acknowledgements}

This work was partly supported by Ministry of Economy, Trade and Industry of Japan and NEDO. We thank Drs. M. Kobashi, S. Kawamura and M. Watanabe for their collaboration.

\section{References}

1. For a review, see C. D. Dimitrakopoulos and D. 
J. Mascaro, IBM J. Res. Dev. 45 (2001) 11.

2. Z. Bao, A. J. Lovinger, and A. Dodabalapur, Appl. Phys. Lett. 69 (1996) 4018.

3. H. Sirringhaus, N. Tessler, R. H. Friend, Science 288, 1741 (1998).

4. H. Sirringhaus, P. J. Brown, R. H. Friend, M. M Nielsen, K. Bechgaard, B.M.W. Langeveld-Voss, A. J. H. Spiering, R. A. J. Janssen, E. W. Meijer, P. Herwig, and D. M. de Leeuw, Nature (London) 401 (1999) 685.

5. M. Raja, G. Lloyd, N. Sedghi, R. di Lucrezia, S. J. Higgins, and W. Eccleston, Mat. Res. Soc. Symp. Proc. 708 (2002) BB10.56.1.

6. G. Wang, J. Swensen, D. Moses, and A. J. Heeger, J. Appl. Phys. 93 (2003) 6137.

7. R. J. Kline, M. D. McGehee, E. N. Kadnikova, J. Liu, and J. M. J. Fréchet, Adv. Mater. 15 (2003) 1519.
8. A. Babel and S. A. Jenekhe, J. Phys. Chem. B 107 (2003) 1749.

9. S. Hoshino, M. Yoshida, S. Uemura, T. Kodzasa, N. Takada, T. Kamata, and K. Yase, $J$. Appl. Phys. in press.

10. S. Hoshino, T. Kamata, and K. Yase, J. Appl. Phys. 92 (2002) 6028.

11. S. M. Sze, "Physics of Semiconductor Devices, 2nd ed", Wiley, New York, (1981) p442.

12. H. Sirringhaus, N. Tessler, D. S. Thomas, P. J.

Brown, R. H. Friend, "Advances in Solid State Physic", vol 39, Vieweg, Braunschweig, Germany, (1999) p101.

13. H. M. Shur, H. Hack, and J. G. Shaw, J. Appl. Phys. 66 (1989) 3371.

14. L. Bürgi, T. J. Richards, R. H. Friend, and H. Sirringhaus, J. Appl. Phys. 94 (2003) 6129. 